\title{
Editorial: Beauty 2018 in La Biodola, Isola d' Elba, Italy
}

\author{
Robert Fleischer \\ Nikhef and Department of Physics and Astronomy, Vrije Universiteit Amsterdam, Amsterdam, \\ Netherlands
}

\section{Neville Harnew, Guy Wilkinson}

Department of Physics, University of Oxford, Oxford, United Kingdom

\section{Fabrizio Palla, Giovanni Punzi}

Sezione INFN di Pisa, Università di Pisa, Pisa, Italy

\begin{abstract}
The International Conference on B-Physics at Frontier Machines, "Beauty 2018", was held in La Biodola, Elba Island, Italy from $6^{\text {th }}$ to $11^{\text {th }}$ May 2018, organized by INFN-Pisa. It was the $17^{\text {th }}$ edition of the series "International Conference on B-Physics at Frontier Machines", initiated in the Czech Republic in 1993. The aims of the conference are to review the latest theoretical and experimental results in heavy flavour physics and to discuss the future programmes. Beauty 2018 covered a wide range of topics including studies of $\mathrm{CP}$ violation and rare decay properties of beauty and charm hadrons. The conference attracted around 80 scientists from all over the world, with a programme consisting of 59 invited talks of which 13 were theoretical topics. In addition, nine early career researchers presented posters.
\end{abstract}

The International Conference on B-Physics at Frontier Machines - BEAUTY2018

6-11 May, 2018

La Biodola, Elba Island, Italy 
These are exciting times for flavour physics, with Run 2 of the LHC providing a wealth of new experimental data and theorists making great efforts to interpret them. Preparations are also well underway towards the LHC experiment upgrades, and Belle-II is now recording its first data. It was therefore no surprise that Beauty 2018 focussed on recent measurements at the high-precision frontier and the quest for physics beyond the Standard Model (SM). The opening presentation was by Matthias Neubert (Johannes Gutenberg University, Mainz), who gave an overview of the theory landscape. Heavy flavour decays, in particular those of $b$-hadrons, offer powerful probes of physics beyond the SM; new particles may manifest themselves in observables which can be calculated and measured with high precision. In recent years several puzzling anomalies have emerged from $\mathrm{LHCb}$ and B-factory data, and discussion of these set the scene for a very inspiring atmosphere at the conference.

Several presentations were focused on the flavour anomalies. In particular the ratio of branching fractions $R\left(D^{(*)}\right)=\mathrm{BR}\left(B \rightarrow D^{(*)} \tau \nu\right) / \mathrm{BR}\left(B \rightarrow D^{(*)} \ell v\right)$ where $\ell=\mu, e$, first measured by Babar and Belle, provide a test of lepton universality, and intriguingly now give combined experimental values which are about $4 \sigma$ away from expectations. Furthermore, the ratios $R(K)=$ $\mathrm{BF}\left(B^{+} \rightarrow K^{+} \mu^{+} \mu^{-}\right) / \mathrm{BF}\left(B^{+} \rightarrow K^{+} e^{+} e^{-}\right)$and the corresponding $K^{*}$ measurement, $R\left(K^{*}\right)$, yield results which are each around $2.5 \sigma$ away from unity. Other potential deviations from the SM are seen in the observable $P_{5}^{\prime}$ of the angular distribution of decay products in the rare decay $B^{0} \rightarrow K^{* 0} \mu^{+} \mu^{-}$, and also measurements in related decay channels, notably in $B^{+} \rightarrow K^{+} \mu^{+} \mu^{-}$, $B_{s} \rightarrow \phi \mu^{+} \mu^{-}$and $\Lambda_{b} \rightarrow \Lambda \mu^{+} \mu^{-}$. Hence the release of the new LHCb results from Run 2 is eagerly awaited later this year.

Observation of the rare decay $B_{s} \rightarrow \mu^{+} \mu^{-}$had already been presented at the $6 \sigma$ level two years ago by a combined analysis of CMS and LHCb data. LHCb now report an observation at the $>5 \sigma$ level in a single experiment, consistent with the Standard Model. The effective lifetime of the decay will offer new ways in the future to search for New Physics, and a first measurement has now been made, $2.04 \pm 0.44 \pm 0.05 \mathrm{ps}$, also consistent with the SM but with large uncertainties.

In addition there were theoretical overview talks, covering topics from uncertainties of key observables within the SM, to scenarios for New Physics. In the context of the flavour anomalies, models with leptoquarks and extra $Z^{\prime}$ bosons are currently receiving much attention. Leptoquarkmediated virtual interactions are currently the preferred mechanism iff the reported flavour anomalies are to be explaining in their entirety. There continues to be impressive progress on lattice QCD calculations and understanding of hadronic form factors which are crucial as inputs for theoretical predictions. Continued interplay between theory and experiment will be essential to understand the emerging data from the LHC and Belle-II.

Concerning $\mathrm{CP}$ violation in the $\mathrm{b}$ sector, $\mathrm{LHCb}$ reported a new world-best determination of the angle $\gamma$ of the unitarity triangle from a combination of measurements, $\left(74.0_{-5.8}^{+5.0}\right)^{\circ}$. A $2 \sigma$ difference from the prediction from other unitarity triangle constraints needs to be monitored. Regarding $\mathrm{CP}$ violation in $B_{s}^{0} \rightarrow J / \psi \phi$ decays, which is predicted to be very small in the SM, the experimental knowledge from a combination of LHC experiments has reached $\phi_{s}=21 \pm 31 \mathrm{mrad}$, which is compatible with the Standard Model at the current level of precision.

There was also a session dedicated to charm physics, focusing on mixing and $\mathrm{CP}$ violation as well as rare $D$-meson decays. Here Belle, BES-III and LHCb showed new results, with precision from the latter experiment recently improved by an order of magnitude. Whilst $\mathrm{CP}$ violation in the 
charm sector has not yet been observed, the SM expectations will soon be approached. BES-III reported new measurements on lepton flavour unitarity in the charm sector, seeing a $1.9 \sigma$ deviation from unity in $D^{+} \rightarrow \tau^{+} v$ when compared with $D^{+} \rightarrow \mu^{+} v$.

Presentations were also devoted to hadron spectroscopy and exotic states, where there has been huge interest since the recent discovery of pentaquark-like states by LHCb. Theoretical models still conjecture whether this is a "deuteron-like" molecular state, or a bound 5-quark object. The (udsb) state reported by D0 over two years ago has not been confirmed in LHC data, and significantly, neither now by CDF. There was a plethora of other new results reported, including from LHCb a doubly charmed baryon, the $\Xi_{c c}^{++}$, and a $\Xi_{b}^{* *-}$ state, as well as a spectroscopy "gold mine" of $X, Y$ and $Z$ states from BES-III.

Kaon physics was also discussed. With the completion of 2016 data analysis, the NA62 experiment has reached SM sensitivity for the ultra-rare $K^{+} \rightarrow \pi^{+} v \bar{v}$ decay channel. A single candidate event was found with 0.15 background events expected, and a lower limit on the branching ratio of $14 \times 10^{-10}$ at $95 \%$ confidence has been set. The analysis of the remaining NA62 data-set and the new run starting in 2021, together with results from the KOTO experiment in Japan, are eagerly anticipated.

Finally, the future experimental programme of flavour physics was discussed at length. One of the highlights of the conference was the report of first data from the Belle-II experiment at the super- $B$ factory at KEKB. Within two years, it is expected that Belle II will collect between 5 to $10 \mathrm{ab}^{-1}$, and will be able to perform very competitive and complementary flavour measurements, including further precision measurements of $R(D)$ and $R\left(D^{*}\right)$ and $B^{0}$ decay channels involving $\tau$ leptons. Further exciting options will also emerge beyond 2021 at the LHC, with LHCb running at an increased luminosity of $2 \times 10^{33} \mathrm{~cm}^{-2} \mathrm{~s}^{-1}$ with an improved trigger, and the high-luminosity ATLAS and CMS upgrades coming in LHC Long-Shutdown 3. The conference was concluded by an excellent and stimulating summary talk from Vincenzo Vagnoni (INFN, Università di Bologna).

The scientific programme was complemented by a variety of social events, including a cocktail party overtooking the Mediterranean Sea, and guided tours of the medieval towns of Capoliveri and Portoferraio. These events, coupled with the stimulating presentations, made the conference a huge success. The detailed programme and presentations are available at the conference web site: https://agenda.infn.it/conferenceDisplay.py?confId=13986. 


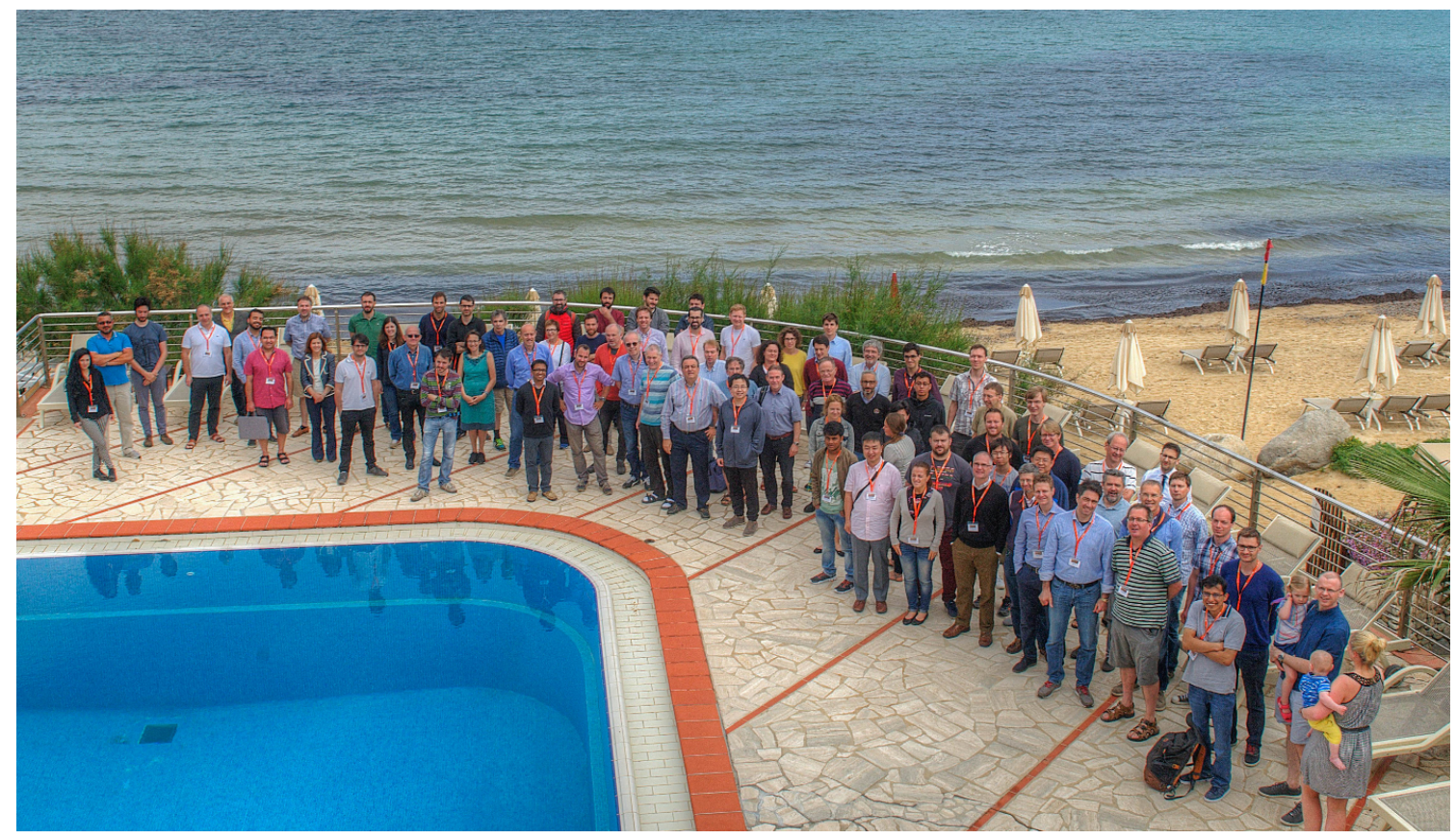

Figure 1: A group photo of the Beauty 2018 participants enjoying a break in proceedings. 